Article

\title{
The Association between Diabetes and Human T-Cell Leukaemia Virus Type-1 (HTLV-1) with Strongyloides stercoralis: Results of a Community-Based, Cross-Sectional Survey in Central Australia
}

\author{
Mohammad Radwanur Talukder ${ }^{1}$ (D) , Hai Pham ${ }^{1}$ (D), Richard Woodman ${ }^{2}$ (D) Kim Wilson ${ }^{3}$, Kerry Taylor ${ }^{4}$, \\ John Kaldor ${ }^{5}$ and Lloyd Einsiedel 1,6,*
}

check for updates

Citation: Talukder, M.R.; Pham, H.; Woodman, R.; Wilson, K.; Taylor, K.; Kaldor, J.; Einsiedel, L. The Association between Diabetes and Human T-Cell Leukaemia Virus Type-1 (HTLV-1) with Strongyloides stercoralis: Results of a Community-Based, Cross-Sectional Survey in Central Australia. Int. J. Environ. Res. Public Health 2022, 19, 2084. https://doi.org/10.3390/ ijerph19042084

Academic Editor: Paul B Tchounwou

Received: 20 December 2021 Accepted: 7 February 2022 Published: 13 February 2022

Publisher's Note: MDPI stays neutral with regard to jurisdictional claims in published maps and institutional affiliations.

Copyright: (C) 2022 by the authors. Licensee MDPI, Basel, Switzerland. This article is an open access article distributed under the terms and conditions of the Creative Commons Attribution (CC BY) license (https:// creativecommons.org/licenses/by/ $4.0 /)$
1 Baker Heart and Diabetes Institute, Alice Springs Hospital, Alice Springs, NT 0870, Australia; radwan.talukder@baker.edu.au (M.R.T.); honghai.pham.vn@gmail.com (H.P.)

2 Flinders Centre for Epidemiology and Biostatistics, Flinders University, Adelaide, SA 5001, Australia; richard.woodman@flinders.edu.au

3 National Serology Reference Laboratory, Melbourne, VIC 3065, Australia; kimmwilson23@gmail.com

4 Poche Centre for Indigenous Health and Wellbeing, Alice Springs, NT 0870, Australia; kerry.taylor@flinders.edu.au

5 Kirby Institute, University of New South Wales, Sydney, NSW 2052, Australia; jkaldor@kirby.unsw.edu.au

6 Alice Springs Hospital, Alice Springs, NT 0870, Australia

* Correspondence: lloyd.einsiedel@nt.gov.au

\begin{abstract}
In central Australia, an area that is endemic for the human T-cell leukaemia virus type-1 (HTLV-1), the prevalence of Strongyloides stercoralis and its association with other health conditions are unknown. A cross-sectional community-based survey was conducted in seven remote Aboriginal communities in central Australia, from 2014 to 2018. All residents aged $\geq 10$ years were invited to complete a health survey and to provide blood for Strongyloides serology, HTLV-1 serology and HTLV-1 proviral load (PVL). Risk factors for Strongyloides seropositivity and associations with specific health conditions including diabetes and HTLV-1 were determined using logistic regression. Overall Strongyloides seroprevalence was 27\% (156/576) (children, $22 \%$ (9/40); adults ( $\geq 15$ years), 27\% $(147 / 536)$, varied widely between communities (5-42\%) and was not associated with an increased risk of gastrointestinal, respiratory or dermatological symptoms. Increasing age, lower HTLV-1 PVL ( $<1000$ copies per $10^{5}$ peripheral blood leucocytes) compared to the HTLV-1 uninfected group and community of residence were significant risk factors for Strongyloides seropositivity in an adjusted model. A modest reduction in the odds of diabetes among Strongyloides seropositive participants was found (aOR 0.58, 95\% CI 0.35, 1.00; $p=0.049$ ); however, this was lost when body mass index was included in the adjusted model (aOR $0.48,95 \%$ CI $0.48,1.47 ; p=0.542$ ). Strongyloides seropositivity had no relationship with anaemia. Exploring social and environmental practices in communities with low Strongyloides seroprevalence may provide useful lessons for similar settings.
\end{abstract}

Keywords: Strongyloides; HTLV-1; diabetes; anaemia; Australia

\section{Introduction}

Infection with the soil-transmitted helminth Strongyloides stercoralis is highly prevalent in tropical and subtropical regions of the world, including Australia [1,2]. Globally, an estimated 370 million people are infected with Strongyloides stercoralis [3]. In Australia, Strongyloides is endemic across the tropical north of the country where prevalence rates range from 10 to $60 \%$ [2]. The nematode has a complicated lifecycle in which rhabditiform larvae excreted in human faeces develop into infective filariform larvae capable of entering the human host by penetrating intact skin. Larvae then enter the venous blood and migrate to the small intestine by way of the lungs [4]. In contrast to other nematodes that infect 
humans, lifelong infestation can be maintained through an auto-infective life cycle in which larvae penetrate the intestinal mucosa [4].

Strongyloides infection can cause diverse symptoms, which reflect the organs involved in the nematode's life cycle. These include pruritus, gastrointestinal symptoms, such as abdominal pain and diarrhoea, and respiratory symptoms, such as cough and dyspnoea $[5,6]$. Immunocompromised individuals, particularly those treated with corticosteroids or infected with the human T-cell leukaemia virus type 1 (HTLV-1) [5,7-9], may be unable to control the larval burden $[8,9]$, resulting in larval migration to the lungs and dissemination to organs, such as the central nervous system, which are not ordinarily affected by larval migration [8,9]. Larval penetration of the gut mucosa in such circumstances may result in life-threatening invasive infections with enteric bacteria [5]. Both corticosteroid therapy and HTLV-1 infection result in an impaired Thelper type 2 (Th2) immune response, which is responsible for controlling many helminth infections including Strongyloides [10]. This is accompanied by downregulated mast cell degranulation, reduced eosinophil recruitment and impaired parasite-killing activity in those infected with HTLV-1 [7]. Consequently, the number of surviving larvae is increased in these individuals, and this increases the risk of hyperinfection and dissemination [7]. HTLV-1 infection has been associated with two-fold increased risk of symptomatic strongyloidiasis compared to patients who are HTLV-1 uninfected [11]. Strongyloides coinfection of people with HTLV-1 induces polyclonal expansion of HTLV-1-infected cells by activating the IL-2/IL-2R system, which could increase the risk of developing HTLV-1 associated diseases [12].

In a central Australian case series, seven of eleven patients with complicated strongyloidiasis were HTLV-1 seropositive, and four died [5]. HTLV-1 co-infection is also associated with the failure of treatment for strongyloidiasis and earlier progression to fatal adult T-cell leukemia $[13,14]$. Reduced efficacy of anthelmintic drugs against Strongyloides is a consequence of impaired Th- 2 immune response, which is modulated by the high IFN- $\gamma$ levels observed in HTLV-1 infected individuals [7]. Treatment failure with anthelmintic drugs may, therefore, be indicative of HTLV-1 infection [2]. A higher number of HTLV-1 infected cells in peripheral blood (HTLV-1 proviral load, PVL) is associated with a higher larval burden as defined by the presence of Strongyloides larvae in stool [15]. Collecting and processing stool samples is challenging in remote areas and strongyloides serology is increasingly used in this setting. Whether higher HTLV-1 PVL are associated with an increased risk of symptomatic strongyloidiasis when this is defined by strongyloides seropositivity has not been studied.

The endemic areas of HTLV-1 and Strongyloides stercoralis overlap in Australia [2]. Relative to non-Indigenous Australians, Australian Aboriginal communities are disproportionately affected by both pathogens. The adult HTLV-1 prevalence in central Australia is the highest reported worldwide and approaches $40 \%$ [16], while the prevalence of strongyloides ranges from 21 to $60 \%$ in the tropical north of Australia [2,4,17]. Crowded and low-quality housing with inadequate sanitation underlie these high rates of infection with Strongyloides [1]. Notwithstanding the extremely high HTLV-1 prevalence in the arid interior of Australia [16] and the increased risks that strongyloides coinfection poses to people with HTLV-1, the epidemiology and clinical manifestations of Strongyloides have been rarely studied in central Australia. Community-based Strongyloides seroprevalence data have so far only been available from a single small community where 10 of 72 adults were Strongyloides seropositive $[2,18]$. Community-based data are needed to support the development of prevention and control strategies for strongyloidiasis in these communities [6].

A higher admission rate with strongyloidiasis was reported among adults with HTLV1 in a retrospective cohort study in central Australia [19]. However, this finding may have been confounded by increased testing and treatment of patients known to be HTLV-1 infected [19]. Recent studies also suggest interactions between Strongyloides and other conditions. For example, strongyloidiasis was associated with an increased risk of anaemia in Ethiopia [20], and strongyloides seropositivity was associated with a reduced risk of diabetes among Aboriginal Australians who attended a remote health clinic [21]. 
We therefore collaborated with remote Aboriginal communities to determine whether Strongyloides seropositivity was associated with HTLV-1 infection and other health conditions, including diabetes and anaemia, in central Australia.

\section{Materials and Methods}

\subsection{Study Setting and Population}

Central Australia is an area of some 1,000,000 km² in which most Aboriginal people live in isolated communities. English is generally the second or third language spoken in these communities. Residents of 7 remote central Australian Aboriginal communities (estimated resident population >15 years, 1153) [22] (designated alphabetically, A to G) participated in this cross-sectional study between August 2014 and June 2018. All communities were extremely disadvantaged as reflected by multiple socio-economic indicators, including median total family income per week (range AUD 492-840), average household size (range 3.7-5.2 members), low proportions of school attendance (range 19-40.3\%), low levels of completed year 12 (range 4.3-9.4\%), and high levels of unemployment (range $19-40.3 \%)[22,23]$.

Details of community engagement and the method of recruitment have been described previously [16]. Briefly, all Aboriginal community residents who were old enough to provide blood via venipuncture (generally $\geq 10$ years) were eligible for recruitment. Participation was voluntary and informed written consent was obtained from all participants prior to commencement of health examinations and sample collections. Consent for children and adolescents ( $<18$ years) was obtained from their legal guardians. Information about the project and for consent was provided in primary Aboriginal languages. A central survey site, usually the primary health care (PHC) clinic, was established in each community. Data from one community have been published previously [18].

\subsection{Data and Specimen Collection}

Participants were asked to complete a simple health questionnaire that recorded alcohol and tobacco consumption, and symptoms classified as respiratory (cough, wheeze and dyspnoea), dermatological (pruritus and rash) or gastrointestinal (abdominal pain and diarrhoea) [24]. The health survey was conducted by a specialist physician qualified in internal medicine and infectious diseases (LE) who was blinded to HTLV-1 and Strongyloides serostatus. An interpreter fluent in local primary languages assisted where necessary. Height and weight were recorded for a concurrent study of spirometry in settings where space could be provided by the participating PHC clinic [21].

Clinic records for five years prior to recruitment were subsequently reviewed for any evidence of comorbid conditions including smoking, harmful alcohol consumption, diabetes, hypertension, chronic kidney disease (CKD) and chronic liver disease. A diagnosis of diabetes was recorded if this was either stated in the medical record or a diabetic medication was prescribed.

Whole blood was collected from participants by venepuncture into EDTA tubes. Peripheral blood buffy coats (PBBC) and plasma were prepared and stored at $-80{ }^{\circ} \mathrm{C}$ at Baker Heart and Diabetes Central Australia (Baker Institute) in Alice Springs.

\subsection{Strongyloides Serology}

Blood samples for Strongyloides serology were collected in gold top serum separator tubes (SST) and stored at $4{ }^{\circ} \mathrm{C}$ at the Baker Institute, Alice Springs. Samples were shipped to Western Diagnostic Pathology, Perth, for Strongyloides serology testing using a commercial IgG enzyme-linked immunosorbent assay (EIA-4208, DRG Instruments, GmbH, Germany), which detects IgG directed against the soluble fraction of filariform Strongyloides stercoralis larvae, in accordance with the manufacturer's protocol. 


\subsection{Haematological Indices}

Point of care tests (HemoCue ${ }^{\circledR}$ WBC DIFF System and Hemocue Hb 301 system, Ängelholm, Sweden) were used to determine haemoglobin concentrations and the total and differential (neutrophils, lymphocytes, monocytes, eosinophils and basophils) white blood cells counts in peripheral blood. Peripheral blood eosinophilia was defined as $>0.6$ cells $/ 10^{9} / \mathrm{L}$.

\subsection{HTLV-1 Tests}

Buffy coats and plasma were shipped to the National Serology Reference Laboratory (NRL), Melbourne, in batches at intervals during the study. HTLV-1 status was confirmed by Western blot analysis (WB) (HTLV-I/II Blot 2.4, MP Diagnostics) on samples reactive on screening either by enzyme immunoassay (Murex HTLV-I + II, DiaSorin, Italy) or particle agglutination assay (Serodia HTLV-1, Fujirebio, Tokyo, Japan). HTLV-1 PVL was determined by polymerase chain reaction (PCR) using DNA extracted from PBBC cells. Primers and probes that targeted a highly conserved region at the $5^{\prime}$ end of the gag gene in the p19 coding region of HTLV-1c (Mel5; accession number, L02534) were expressed as HTLV-1 copies per 105 peripheral blood leucocytes (PBL). Consistent with previous studies [25], HTLV-1 PVL was stratified as low ( $<1000$ copies per $\left.10^{5} \mathrm{PBL}\right)$ and high $(\geq 1000$ copies per $10^{5}$ PBL). The lower limit of detection was 6.5 copies for HTLV-1 (95\% confidence interval [CI], 5.4-8.4) [18]. HTLV-1 infection was defined as a positive WB test or a positive HTLV-1 PCR test.

\subsection{Ethics}

The study was approved by the Central Australian Human Research Ethics Committee (HREC-14-242, 15-322, 16-384).

\subsection{Statistical Analysis}

For categorical variables, frequency and percentages were used; for continuous variables, mean, standard deviation (SD), and median were used. Strongyloides seropositive and seronegative groups were compared using Pearson's $\chi^{2}$ test, Student's $t$-test or MannWhitney test as appropriate for each variable. A multivariate logistic regression model was used to determine the factors associated with Strongyloides seropositivity (age, sex, residence, tobacco use, alcohol consumption, diabetes, chronic kidney disease stages 2-5, chronic liver disease, and HTLV-1 seropositivity). The odds ratio with $95 \%$ confidence intervals $(\mathrm{CI})$ and $p$-value $(<0.05)$ were used to report the associations between Strongyloides seropositivity and other variables. All analyses were conducted in Stata version14 (StataCorp, College Station, TX, USA).

\section{Results}

Strongyloides serology was tested for 576 participants, approximately $37.5 \%$ of the estimated resident population (children under 15, $40 / 169$ (24\%); adults ( $\geq 15$ years), 536/1153 $(46 \%))$. Overall Strongyloides seropositivity rates for children and adults were $22 \%(9 / 40)$ and $27 \%(147 / 536)$, respectively.

Overall mean age of adults did not differ between Strongyloides seropositive (Strongyloides + ve) and seronegative (Strongyloides - ve) groups (Table 1). However, Strongyloides seroprevalence was highest among the 45-54 years group (40\%) and lowest among the 35-44 years group (18\%) (Table 1). Strongyloides seropositivity differed considerably between communities, ranging from $5 \%$ in community $\mathrm{C}$ to $42 \%$ in Community $\mathrm{A}$ and Community G $(p<0.001)$ (Table 1$)$.

\subsection{Clinical Findings}

Prevalence of gastrointestinal, respiratory and dermatological symptoms did not differ according to Strongyloides serostatus (Table 2). 
Table 1. Demographic characteristics of adult Aboriginal participants by Strongyloides serostatus $(n=536)$.

\begin{tabular}{cccc}
\hline Characteristics & $\begin{array}{c}\text { Strongyloides }- \text { ve } \\
(\boldsymbol{n}=\mathbf{3 8 9 )}\end{array}$ & $\begin{array}{c}\text { Strongyloides +ve } \\
(\boldsymbol{n}=\mathbf{1 4 7})\end{array}$ & $\boldsymbol{p}$-Value \\
\hline Demographic & & & \\
\hline Age (Mean (sd)) & $38.0(14.7)$ & $40.4(15.4)$ & 0.108 \\
Age groups (years) (n (\%)) & $79(73.8)$ & $28(26.2)$ & \\
15-24 years & $95(75.4)$ & $31(24.6)$ & \\
25-34 years & $91(82.0)$ & $20(18.0)$ & \\
35-44 years & $68(60.2)$ & $45(39.8)$ & \\
45-54 years & $56(70.9)$ & $23(29.1)$ & \\
$\geq 55$ years & & $66(27.0)$ & \\
Sex (n (\%)) & $178(72.9)$ & $81(27.7)$ & \\
Male & $211(72.3)$ & $34(42.0)$ & \\
Female & & $23(32.4)$ & \\
Residence (n (\%)) & $47(58.0)$ & $6(5.4)$ & \\
Community A & $48(67.6)$ & $54(36.7)$ & \\
Community B & $105(94.6)$ & $9(14.7)$ & \\
Community C & $93(63.3)$ & $7(21.9)$ & \\
Community D & $52(85.3)$ & $14(42.4)$ & \\
Community E & $25(78.1)$ & $19(57.6)$ &
\end{tabular}

Table 2. Health-related conditions of adult Aboriginal participants by Strongyloides serostatus $(n=536)$.

\begin{tabular}{|c|c|c|c|}
\hline $\begin{array}{l}\text { Health Related Conditions }{ }^{a} n \\
(\%)\end{array}$ & $\begin{array}{l}\text { Strongyloides }- \text { ve } \\
\quad(n=389)\end{array}$ & $\begin{array}{l}\text { Strongyloides +ve } \\
\quad(n=147)\end{array}$ & $p$-Value \\
\hline Diabetes & & & 0.294 \\
\hline Yes & $146(75.3)$ & $48(24.7)$ & \\
\hline No & $243(71.1)$ & $99(28.9)$ & \\
\hline Hypertension & & & 0.548 \\
\hline Yes & $96(70.6)$ & $40(29.4)$ & \\
\hline No & $293(73.2)$ & $107(26.7)$ & \\
\hline $\mathrm{CKD}^{\mathrm{b}}$ & & & 0.724 \\
\hline Yes & $100(71.4)$ & $40(28.6)$ & \\
\hline No & $289(73.0)$ & $107(27.0)$ & \\
\hline Liver disease & & & 0.419 \\
\hline Yes & $15(65.2)$ & $8(34.8)$ & \\
\hline No & $374(72.9)$ & $139(27.1)$ & \\
\hline Smoking & & & 0.068 \\
\hline Yes & $209(76.0)$ & $66(24.0)$ & \\
\hline No & $180(69.0)$ & $81(31.0)$ & \\
\hline Alcohol $^{\mathrm{c}}$ & & & 0.779 \\
\hline Yes & $164(72.1)$ & $60(21.9)$ & \\
\hline No & $225(73.2)$ & $87(26.8)$ & \\
\hline \multicolumn{4}{|l|}{ Clinical history/examinations } \\
\hline GI symptoms $\mathrm{d}$ & & & 0.252 \\
\hline Yes & $16(64.0)$ & $9(36.0)$ & \\
\hline No & $299(74.4)$ & $103(25.6)$ & \\
\hline Resp symptoms e & & & 0.924 \\
\hline Yes & $49(74.2)$ & $17(25.8)$ & \\
\hline No & $266(73.7)$ & $95(26.3)$ & \\
\hline
\end{tabular}


Table 2. Cont.

\begin{tabular}{|c|c|c|c|}
\hline $\begin{array}{l}\text { Health Related Conditions }{ }^{a} n \\
(\%)\end{array}$ & $\begin{array}{l}\text { Strongyloides - ve } \\
\qquad(n=389)\end{array}$ & $\begin{array}{l}\text { Strongyloides +ve } \\
\quad(n=147)\end{array}$ & $p$-Value \\
\hline Skin symptoms ${ }^{\mathrm{f}}$ & & & 0.381 \\
\hline Yes & $15(65.2)$ & $8(34.8)$ & \\
\hline No & $248(73.6)$ & $89(26.4)$ & \\
\hline BMI g (Mean (sd)) & $28.9(6.9)$ & $28.4(5.5)$ & 0.511 \\
\hline \multicolumn{4}{|l|}{ Blood results } \\
\hline HTLV-1 h & & & 0.043 \\
\hline Yes & $156(77.6)$ & $45(22.4)$ & \\
\hline No & $233(69.5)$ & $102(30.5)$ & \\
\hline \multicolumn{4}{|l|}{ HTLV-1 PVL i } \\
\hline Uninfected & $233(69.5)$ & $102(30.5)$ & 0.101 \\
\hline HTLV-1 low PVL & $103(79.2)$ & $27(20.8)$ & \\
\hline HTLV-1 high PVL & $53(74.6)$ & $18(25.4)$ & \\
\hline
\end{tabular}

a Health related conditions identified from medical records. These included diabetes, hypertension, chronic kidney disease (CKD stage 1 and above), and liver disease other than chronic hepatitis B virus, smoking and harmful alcohol consumption. ${ }^{\mathrm{b}}$ CKD-Chronic Kidney Disease, CKD Stage 1 and above. ${ }^{\mathrm{c}}$ Harmful alcohol consumption documented in medical records. ${ }^{\mathrm{d}}$ Gastrointestinal symptoms $(n=427)$ include abdominal pain and diarrhoea. Diarrhoea was defined as loose stools, $>3$ times per day for $>2$ days in the preceding week e Respiratory symptoms $(n=427)$ include cough, wheeze and breathing problem. ${ }^{\mathrm{f}}$ Skin problems $(n=360)$ include pruritus and skin rash excluding scabies. 9 BMI-Body mass index as $\mathrm{kg}$ per $\mathrm{m}^{2}, n=379$, from clinical examination. ${ }^{\mathrm{h}}$ HTLV-1 infection was determined by Western blot in 193 subjects and by PCR in 8 subjects with indeterminate WB. ${ }^{i}$ Low and high HTLV-1 PVL were HTLV-1 PVL $<1000$ and $\geq 1000$ copies per 105 peripheral blood leukocytes, respectively.

Though still in the normal range, neutrophil counts were significantly lower and eosinophil counts were significantly higher among Strongyloides seropositive adults (Table 3). There were no differences in haematological parameters among children according to Strongyloides serostatus (Table 3). No significant difference in mean haemoglobin level between Strongyloides seropositive and seronegative groups was observed for either adults or children (Table 3).

Table 3. Haematological parameters according to Strongyloides serostatus for adults and children.

\begin{tabular}{|c|c|c|c|c|c|c|}
\hline \multirow{2}{*}{$\begin{array}{l}\text { Blood Results } \\
\text { (Median } \\
\text { [Range]) }^{a}\end{array}$} & \multicolumn{3}{|c|}{$\begin{array}{l}\text { Adults } \\
n=446\end{array}$} & \multicolumn{3}{|c|}{$\begin{array}{c}\text { Children } \\
n=31\end{array}$} \\
\hline & $\begin{array}{c}-\mathrm{ve}^{\mathrm{b}} \\
n=327\end{array}$ & $\begin{array}{c}+\mathrm{ve}^{\mathrm{c}} \\
n=119\end{array}$ & $p$-Value ${ }^{\mathrm{d}}$ & $\begin{array}{l}-\mathrm{ve}^{\mathrm{b}} \\
n=26\end{array}$ & $\begin{array}{l}+\mathrm{ve}^{\mathrm{c}} \\
n=5\end{array}$ & $p$-Value ${ }^{\mathrm{d}}$ \\
\hline $\begin{array}{l}\text { Haemoglobin } \\
\qquad \begin{array}{c}(\mathrm{g} / \mathrm{L}) \\
(n=441)\end{array}\end{array}$ & $\begin{array}{c}138 \\
{[44-192]}\end{array}$ & $\begin{array}{c}139 \\
{[89-217]}\end{array}$ & 0.602 & $\begin{array}{c}127 \\
{[68-165]}\end{array}$ & $\begin{array}{c}136 \\
{[113-165]}\end{array}$ & 0.608 \\
\hline $\begin{array}{l}\text { White Blood } \\
\text { Cells }\left(10^{9} / \mathrm{L}\right)\end{array}$ & $\begin{array}{c}8.6 \\
{[3.6-18.8]}\end{array}$ & $\begin{array}{c}8.4 \\
{[3.2-18.6]}\end{array}$ & 0.094 & $\begin{array}{c}8.5 \\
{[4.4-14.0]}\end{array}$ & $\begin{array}{c}10 \\
{[4.1-11.7]}\end{array}$ & 0.271 \\
\hline $\begin{array}{c}\text { Neutrophils } \\
\left(10^{9} / \mathrm{L}\right)\end{array}$ & $\begin{array}{c}4.8 \\
{[0.6-15.2]}\end{array}$ & $\begin{array}{c}4.5 \\
{[1.3-13.6]}\end{array}$ & 0.047 & $\begin{array}{c}4.0 \\
{[1.4-8.5]}\end{array}$ & $\begin{array}{c}5.3 \\
{[1.8-5.4]}\end{array}$ & 0.501 \\
\hline $\begin{array}{l}\text { Lymphocytes } \\
\left(10^{9} / \mathrm{L}\right)\end{array}$ & $\begin{array}{c}2.7 \\
{[0.6-8.8]}\end{array}$ & $\begin{array}{c}2.5 \\
{[0.8-5.9]}\end{array}$ & 0.377 & $\begin{array}{c}3.2 \\
{[1.6-6.2]}\end{array}$ & $\begin{array}{c}4.0 \\
{[1.6-4.6]}\end{array}$ & 0.484 \\
\hline $\begin{array}{c}\text { Eosinophils } \\
\left(10^{9} / \mathrm{L}\right)\end{array}$ & $0.2[0-2.7]$ & $0.3[0-2.0]$ & 0.024 & $\begin{array}{c}0.45 \\
{[0.1-1.1]}\end{array}$ & $\begin{array}{c}0.4 \\
{[0.1-1.4]}\end{array}$ & 0.871 \\
\hline
\end{tabular}

a Blood results are presented with Median [range]. ${ }^{\mathrm{b}}-\mathrm{ve}$, Strongyloides seronegative. ${ }^{\mathrm{c}}+\mathrm{ve}$, Strongyloides seropositive. ${ }^{\mathrm{d}} p$ value, based on rank sum test.

\subsection{Strongyloides Seropositivity and Diabetes}

The prevalence of comorbid conditions including diabetes and harmful alcohol consumption did not differ according to Strongyloides seropositivity (Table 2). Diabetes was associated with a modestly lower odds of Strongyloides seropositivity in the adjusted model (aOR 0.58, 95\% CI 0.35, 1.00; $p=0.049)$ (Table 4). Significantly lower odds was also apparent 
for the Strongyloides seropositive group when risk factors for diabetes were analysed separately (aOR 0.59, 95\% CI 0.37, 0.95; $p=0.03$ ) (Table 4); however, there was no association between diabetes and Strongyloides seropositivity when BMI was included in the analysis $(n=379 ; \mathrm{aOR} 0.48,95 \%$ CI $0.48,1.47 ; p=0.542)$ (Table 4$)$.

Table 4. The relationship between Strongyloides seropositivity and diabetes in Aboriginal adults.

\begin{tabular}{|c|c|c|c|c|c|c|c|c|c|c|c|c|}
\hline \multirow[b]{2}{*}{ Diabetes } & \multicolumn{6}{|c|}{ All Participants $(n=536)$} & \multicolumn{6}{|c|}{ Participants with BMI ${ }^{d}(n=379)$} \\
\hline & $\mathbf{u O R}^{\mathrm{a}}$ & $95 \% \mathrm{CI}$ & $p$-Value & $\mathrm{aOR} * \mathrm{~b}$ & $95 \% \mathrm{CI}$ & $p$-Value & $\mathbf{u O R}^{\mathbf{b}}$ & $95 \% \mathrm{CI}$ & $p$-Value & $\mathrm{aOR}^{\mathrm{b} * *}$ & $95 \% \mathrm{CI}$ & $p$-Value \\
\hline Age $^{c}$ (years) & 1.09 & $\begin{array}{l}1.07 \\
1.10\end{array}$ & $<0.001$ & 1.09 & $\begin{array}{l}1.07 \\
1.11\end{array}$ & $<0.001$ & 1.08 & $\begin{array}{l}1.06 \\
1.10\end{array}$ & $<0.001$ & 1.08 & $\begin{array}{l}1.06 \\
1.10\end{array}$ & $<0.001$ \\
\hline Male gender & 0.69 & $\begin{array}{l}0.48 \\
0.98\end{array}$ & 0.042 & 0.79 & $\begin{array}{l}0.50 \\
1.24\end{array}$ & 0.306 & 0.56 & $\begin{array}{l}0.36 \\
0.87\end{array}$ & 0.009 & 0.67 & $\begin{array}{l}0.38 \\
1.17\end{array}$ & 0.125 \\
\hline $\begin{array}{l}\text { Harmful alcohol } \\
\text { consumption }\end{array}$ & 1.48 & $\begin{array}{l}1.04 \\
2.12\end{array}$ & 0.03 & 1.41 & $\begin{array}{l}0.91 \\
2.20\end{array}$ & 0.123 & 1.45 & $\begin{array}{l}0.95 \\
2.23\end{array}$ & 0.087 & 1.51 & $\begin{array}{l}0.90 \\
2.55\end{array}$ & 0.121 \\
\hline Smoking & 0.81 & $\begin{array}{l}0.57 \\
1.15\end{array}$ & 0.24 & 0.94 & $\begin{array}{l}0.59 \\
1.51\end{array}$ & 0.814 & 0.82 & $\begin{array}{l}0.53 \\
1.26\end{array}$ & 0.367 & 1.30 & $\begin{array}{l}0.74 \\
2.28\end{array}$ & 0.369 \\
\hline $\begin{array}{l}\text { Strongyloides } \\
\text { seropositive }\end{array}$ & 0.81 & $\begin{array}{l}0.54 \\
1.20\end{array}$ & 0.295 & 0.59 & $\begin{array}{l}0.37 \\
0.95\end{array}$ & 0.03 & 0.92 & $\begin{array}{l}0.57 \\
1.50\end{array}$ & 0.749 & 0.84 & $\begin{array}{l}0.48 \\
1.47\end{array}$ & 0.542 \\
\hline $\mathrm{BMI}^{\mathrm{d}}\left(\mathrm{kg} / \mathrm{m}^{2}\right)$ & - & - & - & - & - & - & 1.09 & $\begin{array}{l}1.05 \\
1.13\end{array}$ & $<0.001$ & 1.07 & $\begin{array}{l}1.03 \\
1.12\end{array}$ & 0.001 \\
\hline
\end{tabular}

* Adjusted for age, sex, smoking, harmful alcohol consumption. ${ }^{* *}$ Adjusted for age, sex, smoking, harmful alcohol consumption and BMI (body mass index). ${ }^{a}$ unadjusted odds ratio. ${ }^{b}$ Adjusted odds ratio. ${ }^{c}$ Age per year. ${ }^{d}$ BMIBody Mass Index.

\subsection{Strongyloides Seropositivity and HTLV-1}

Strongyloides seroprevalence was higher in the HTLV-1 uninfected group (102/335, $30.4 \%)$ relative to the HTLV-1 infected group $(45 / 201,22.4 \%)(p=0.043)$ (Table 2). When Strongyloides seroprevalence was stratified by HTLV-1 PVL, compared to the HTLV-1 uninfected group, HTLV-1 low PVL (20.8\%) and HTLV-1 high PVL (25.4\%) groups had lower Strongyloides seroprevalence, but it was not statistically significant (Table 2).

In an adjusted model, risk of Strongyloides seropositivity increased with age, and was reduced among participants with a low HTLV-1 PVL compared to HTLV-1 uninfected group (Table 5). Relative to residents of community C, risk of Strongyloides seropositivity was increased three to thirteen-fold among residents in other study communities (Table 5).

Table 5. Risk factors for Strongyloides seropositivity among adult participants $(n=536)$.

\begin{tabular}{|c|c|c|c|c|c|c|}
\hline \multirow[t]{2}{*}{ Characteristics } & \multicolumn{3}{|c|}{ Unadjusted } & \multicolumn{3}{|c|}{ Adjusted $^{a}$} \\
\hline & OR & $95 \%$ CI & $p$-Value & OR & $95 \%$ CI & $p$-Value \\
\hline Age $^{b}$ & 1.01 & 0.997-1.02 & 0.122 & 1.02 & $1.005,1.04$ & 0.01 \\
\hline Male & 0.96 & $0.66,1.41$ & 0.858 & 1.28 & $0.81,2.01$ & 0.291 \\
\hline \multicolumn{7}{|l|}{ Residence } \\
\hline Community $\mathrm{C}$ & ref & & & & & \\
\hline Community A & 12.66 & $4.98,32.20$ & $<0.001$ & 12.74 & $4.94,32.85$ & $<0.001$ \\
\hline Community B & 8.38 & $3.21,21.93$ & $<0.001$ & 7.72 & $2.92,20.39$ & $<0.001$ \\
\hline Community D & 10.16 & $4.18,24.70$ & $<0.001$ & 9.84 & $4.00,24.18$ & $<0.001$ \\
\hline Community E & 3.03 & $1.02,8.96$ & 0.045 & 2.93 & $0.97,8.82$ & 0.056 \\
\hline Community $\mathrm{F}$ & 4.90 & $1.51,15.86$ & 0.008 & 3.89 & $1.16,13.06$ & 0.028 \\
\hline Community G & 12.89 & $4.40,37.74$ & $<0.001$ & 13.07 & $4.36,39.18$ & $<0.001$ \\
\hline Smoking ${ }^{\mathrm{c}}$ & 0.70 & $0.48,1.03$ & 0.069 & 0.75 & $0.47,1.19$ & 0.217 \\
\hline Alcohol $^{\mathrm{d}}$ & 0.95 & $0.64,1.39$ & 0.779 & 0.95 & $0.60,1.52$ & 0.839 \\
\hline Diabetes ${ }^{\mathrm{e}}$ & 0.81 & $0.54,1.20$ & 0.295 & 0.58 & $0.34,1.00$ & 0.049 \\
\hline $\mathrm{CKD}^{\mathrm{e}}$ & 1.08 & $0.70,1.66$ & 0.724 & 1.07 & $0.62,1.84$ & 0.816 \\
\hline $\operatorname{CLD}^{\mathrm{f}}$ & 1.43 & $0.59,3.46$ & 0.421 & 1.55 & $0.58,4.27$ & 0.362 \\
\hline \multicolumn{7}{|l|}{ HTLV-1 status $\mathrm{g}$} \\
\hline Uninfected & ref & & & & & \\
\hline
\end{tabular}


Table 5. Cont.

\begin{tabular}{|c|c|c|c|c|c|c|}
\hline \multirow[t]{2}{*}{ Characteristics } & \multicolumn{3}{|c|}{ Unadjusted } & \multicolumn{3}{|c|}{ Adjusted $^{a}$} \\
\hline & OR & $95 \% \mathrm{CI}$ & $p$-Value & OR & $95 \% \mathrm{CI}$ & $p$-Value \\
\hline HTLV1 low PVL ${ }^{h}$ & 0.60 & $0.37,0.97$ & 0.038 & 0.55 & $0.32,0.95$ & 0.031 \\
\hline HTLV1 high PVL ${ }^{h}$ & 0.77 & $0.43,1.39$ & 0.394 & 0.81 & $0.42,1.56$ & 0.531 \\
\hline
\end{tabular}

${ }^{a}$ model was adjusted for age, sex, residence (community), smoking, alcohol consumption, diabetes, CKD stage 1 ad above, liver disease and HTLV-1 status by PVL. ${ }^{b}$ Age per year. ${ }^{c}$ Smoking status documented in medical records. Reference is no smoking. ${ }^{d}$ Harmful alcohol consumption documented in medical records. Reference is no harmful alcohol consumption. ${ }^{e}$ Diabetes and chronic kidney disease (CKD) identified from medical records. ${ }^{\mathrm{f}}$ CLD—chronic liver disease other than chronic hepatitis B virus. ${ }^{\mathrm{g}}$ HTLV-1 infection was determined by Western blot in 193 subjects and by PCR in 8 subjects with indeterminate WB. ${ }^{\mathrm{h}}$ Low and high HTLV-1 PVL were HTLV-1 PVL $<1000$ and $\geq 1000$ copies per 105 peripheral blood leukocytes, respectively.

\section{Discussion}

In the first study of Strongyloides seroprevalence in remote central Australian communities, and the first to stratify risk according to HTLV-1 PVL, we found an overall prevalence exceeding $20 \%$ for Aboriginal adults and children. The nematode is most prevalent in the tropical and sub-tropical regions of developing countries [1], and high endemicity has long been recognised in remote Aboriginal communities in tropical Australia where prevalence rates may be as high as $60 \%$ [2]. Chronic exposure to Strongyloides reflects a persistent social disadvantage in remote Aboriginal communities in which overcrowded housing and non-functioning health hardware lead to environmental conditions that are detrimental to householders [1]. Although data on health infrastructure were not available, other socio-economic indices were substantially lower than national averages for all study communities $[22,23,26]$. Stronglyoides seroprevalence varied widely between communities in central Australia. Interestingly, the lowest seroprevalence was recorded for community $\mathrm{C}$, which also had the largest households, lowest household income and lowest educational attainment according to ABS and regional data [23,26]. Identifying factors that protect these residents from the high regional prevalence of Strongyloides may contribute to the development of culturally appropriate control strategies elsewhere.

The mass drug administration (MDA) of ivermectin, either alone [27] or in combination with community participation [17] and environmental measures [6], has produced substantial reductions in Strongyloides prevalence in Australia [17,28] and overseas [29]. In one community in the far north of Australia, an ivermectin MDA reduced Strongyloides seroprevalence from $21 \%$ at baseline to $2 \%$ at month 18 [17]. Community ownership and improved sanitation are likely to be necessary for sustained reductions in prevalence [30]. Nevertheless, evidence of adverse outcomes among community residents who are Strongyloides seropositive is lacking and there has been considerable debate as to whether public health strategies to reduce Strongyloides prevalence are warranted in Australia [31], and no global consensus has emerged [32]. Although respiratory (16\%), gastrointestinal ( $8 \%)$ and dermatological symptoms (8\%) were relatively common in our cross-sectional study in Central Australian Aboriginal communities, these were not correlated with Strongyloides seropositivity. Similarly, a case-control study [31] and baseline and follow up clinical assessments for an MDA [17] in northern Australia were unable to find an association between Strongyloides seropositivity and clinical symptoms. Although there is currently no evidence that Strongyloides seropositivity is associated with gastrointestinal, respiratory or dermatological symptoms in remote Aboriginal communities [31] in the NT, correlating Strongyloides seropositivity with uncomplicated chronic strongyloidiasis in a population-based survey is difficult.

Notwithstanding reports that chronic helminth infections have a protective effect on metabolic diseases including reduced risks of hyperglycaemia, type 2 diabetes, metabolic syndrome and insulin resistance [33], the evidence for an association between Strongyloides and diabetes is scarce and remains inconclusive. A reduced risk of diabetes has been reported among Aboriginal Australians with Strongyloides infection in a community clinic 
setting [21] and in a hospital-based study from India [34]. Three principal mechanisms have been proposed to account for this protective effect [33]. A nutrition-based mechanism postulates that low body weight as a consequence of chronic helminth infection could contribute to improved metabolic outcomes [33]. Consistent with previous studies [35], we found no difference in BMI according to Strongyloides seropositivity and there was no association between Strongyloides infection and diabetes in the adjusted multivariate model that included BMI. Alterations to the gut microbiome of the human host are purported to result from helminth infection, and these may reduce the risk of diabetes by modulating glucose uptake, inflammation, and insulin sensitivity $[33,36]$. However, the relationship between helminths including Strongyloides, the microbiome and metabolic outcomes is yet to be fully explored [33].

A stronger case has been made for Strongyloides-mediated immune-modulation of glycaemic, hormonal, and cytokine parameters which may have anti-diabetogenic effects. Significantly reduced insulin and glucagon levels have been reported among Strongyloides infected individuals, which was confirmed by faecal microscopy, compared to those who are uninfected, and these effects reversed after anthelminthic therapy [36]. Strongyloides infection significantly reduces the pro-inflammatory milieu in T2DM by lowering the systemic levels of cytokines and chemokines [37]. Decreased blood insulin levels may result from an imbalance of pro- and anti-inflammatory adipokines. Adiponectin is a major inflammatory modulator that influences glucose homeostasis and insulin resistance in diabetes [38]. Modulation of adipocytokines due to Strongyloides infection may also confer a degree of protection against the severity of type 2 diabetes [36]. Chronic helminth infections mediate a modified Th2 immune response, resulting in reduced pro-inflammatory cytokines, such as IFN- $\gamma$ and TNF- $\alpha$, and an increase in anti-inflammatory cytokines, such as IL-10 and TGF- $\beta$ [36]. An epiphenomenon of this survival strategy is likely to be an overall reduction in systemic inflammation within the host and an increase in insulin sensitivity [21]. This hypothesis is consistent with the low-level chronic inflammation that is a common feature of diabetes [39] and with the negative association between IL-10 and diabetes that has been reported in other settings [38]. Nevertheless, case-control studies from the UK [35] and Brazil [40] found an increased risk of Strongyloides seropositivity among adults with diabetes, and in the first community-based study to address this issue, we found that Strongyloides seropositivity was not associated with diabetes when BMI was included in the multivariate model.

Studying the health interactions of Strongyliodes in the human host is made challenging by the difficulty with which chronic strongyloidiasis is diagnosed and by the potential for Strongyloides serology to remain positive after resolution of infection. Although the identification of larvae in faecal samples by microscopy is considered the 'gold standard' method by which Strongyloides infection is diagnosed [41], the sensitivity of this technique is poor due to low and intermittent larval output in faeces [42]. In contrast, serological tests are highly sensitive, but have a lower specificity than faecal microscopy [43]. A recent systematic review and meta-analysis reported that the sensitivity and specificity of serological techniques relative to faecal microscopy also vary considerably for different tests, ranging from $59.1 \%$ to $90.5 \%$ and $61.8 \%$ to $93.7 \%$, respectively [44]. The ELISA-IgG test that was used for Strongyloides diagnosis in this research has been reported as $92.3 \%$ sensitivity and $97.4 \%$ specificity relative to faecal results [42]. Cross-reactivity with other helminths, such as hookworm, can also result in false-positive results and overestimate the prevalence of Strongyloides [45]; however, this is unlikely to affect our results because nematodes other than Strongyloides are not endemic in central Australia. An underestimation of prevalence may also occur because of false-negative results, especially in acute infections and among immunosuppressed patients [43]. Strongyloides seroprevalence in HTLV-1 endemic areas, such as central Australia, may be underestimated by lower Strongyloides serology titres among individuals with HTLV-1 [2], and this could contribute to the lack of an association between HTLV-1 and Strongyloides seropositivity that is reported here and in other HTLV-1 endemic areas. 
Several studies have reported an association between Strongyloides infection and anaemia [20,46]. In a recent Ethiopian study that included other soil transmitted helminths including hookworm, the presence of Strongyloides larvae in stool was associated with a five-fold increased risk of anaemia [20], although it was unclear whether other helminth infections were included in the adjusted model. No relationship between strongyloidiasis and anaemia was found in a recent systematic review of pregnant women [47]. Confounders such as the precarious socioeconomic conditions in which participants lived, which increased risk of exposure to Strongyloides and to malnutrition, might be the more important cause of anaemia and complicate the interpretation of these studies [47]. In the present study, and in a previous study of childhood strongyloidiasis in central Australia [48], all participants lived in similarly disadvantaged circumstances with ready access to health clinics and there was no association between anaemia and Strongyloides seropositivity.

Higher HTLV-1 PVL increases risk of symptomatic strongyloidiasis when this is defined by faecal microscopy [15], and this inability to control the larval burden may be responsible for higher rates of treatment failure among patients with HTLV-1 infection when treated with thiabendazole, albendazole, or ivermectin $[13,14]$. Our observation that Strongyloides seropositivity rates were lower among participants with low HTLV-1 PVL relative to those with high HTLV-1 PVL was therefore surprising. The effect of HTLV-1 PVL on Strongyloides seropositivity has not been studied previously, and further studies are necessary to understand this finding. However, this might reflect the greater likelihood of treatment for strongyloidiasis among people who are HTLV-1 seropositive and higher rates of treatment failure in those who with high HTLV-1 PVL.

Strengths of the present study are the recruitment of participants in a community setting with blinding of both researchers and participants to Strongyloides and HTLV-1 serostatus. However, some important limitations must be recognised. We recruited less than half of all eligible community members, raising the possibility of selection bias, and the recruitment of participants with better health engagement who may be more likely to receive treatment for strongyloidiasis. This might affect estimates of Strongyloides seroprevalence, and true prevalence may be higher than that reported here. However, selection bias is unlikely to alter the interpretation of our clinical survey, which was blinded to Strongyloides and HTLV-1 serostatus. Second, our clinical findings relate only to Strongyloides seropositivity, which reflects exposure to Strongyloides rather than active strongyloidiasis and has no relationship to larval burden. Third, we found no evidence of an association between Strongyloides seropositivity and gastrointestinal symptoms, which can fluctuate over time and may not be captured in our cross-sectional study. Our inability to obtain faecal samples prevented us from estimating the Strongyloides larval burden and we were also unable to exclude other pathogens, such as giardia and cryptosporidium, which are important causes of gastrointestinal symptoms in our region. Nevertheless, the number of participants with such symptoms was low for both Strongyloides seropositive and seronegative groups. Finally, we were unable to collect data for other important community and environmental factors, such as health engagement and treatment, or behaviours that might increase exposure to Strongyloides, which could explain the variation in prevalence.

\section{Conclusions}

In conclusion, we demonstrate a high Strongyloides seroprevalence in an arid, HTLV-1 endemic region of remote Australia. Consistent with other recent studies from the far north of the NT $[17,31]$, Strongyloides seropositivity was not associated with clinical symptoms in our cross-sectional study in this HTLV-1 endemic area, nor was it associated with diabetes or anaemia. The single case of complicated strongyloidiasis that has been reported from this region since awareness was raised among clinicians a decade ago [5] followed iatrogenic immunosuppression without prior testing for strongyloidiasis [49]. This supports the current approach of testing and treating patients with clinical symptoms along with those with immune deficiencies due to HTLV-1 or iatrogenic immunosuppression [31]. Although the overall Strongyloides seroprevalence in central Australia was high, the wide 
variation in prevalence suggests that some Aboriginal communities may have developed protective strategies that reduce risk of infection with soil-transmitted helminths. An in-depth exploration of social and environmental practices in low-risk communities may provide useful lessons for other remote Aboriginal communities to reduce overall risk of strongyloidiasis.

Author Contributions: Conceptualization, M.R.T. and L.E.; methodology, M.R.T., L.E.; validation, M.R.T., H.P., K.W. and L.E.; formal analysis, M.R.T., R.W.; investigation, K.W.; resources, L.E., K.T.; data curation, M.R.T., H.P.; writing—original draft preparation, M.R.T.; writing—review and editing, M.R.T., R.W., J.K., L.E., K.W.; visualization, M.R.T.; supervision, H.P., L.E.; project administration, H.P., M.R.T., K.T., L.E.; funding acquisition, J.K., L.E. All authors have read and agreed to the published version of the manuscript.

Funding: This project was funded by the National Health and Medical Research Council of Australia (project grant 1088517) (awarded to L.E., K.W. and K.T.). The funder had no role in the study design, data collection and analysis, decision to publish, or manuscript preparation.

Institutional Review Board Statement: The study was conducted according to the guidelines of the Declaration of Helsinki, and approved by the Central Australian Human Research Ethics Committee (HREC-14-242, 15-322, 16-384).

Informed Consent Statement: Informed written consent was obtained from all subjects involved in the study.

Data Availability Statement: These data relate to an Australian Aboriginal population and are culturally sensitive. Although they cannot be shared publicly for ethical reasons, data will be made available to researchers who have received written approval from the Central Australian Human Research Ethics Committee (contact via cahrec@flinders.edu.au).

Acknowledgments: We gratefully acknowledge the contributions made to this project by Aboriginal researchers Clinton Pepperill and Una Ratara who recruited participants, PHC community clinics who generously provided space despite the heavy clinical demands made of them, and the pathology service at Alice Springs Hospital.

Conflicts of Interest: The authors declare no conflict of interest.

\section{References}

1. Beknazarova, M.; Whiley, H.; Ross, K. Strongyloidiasis: A Disease of Socioeconomic Disadvantage. Int. J. Environ. Res. Public Health 2016, 13, 517. [CrossRef]

2. Gordon, C.A.; Shield, J.M.; Bradbury, R.S.; Muhi, S.; Page, W.; Judd, J.A.; Lee, R.; Biggs, B.A.; Ross, K.; Kurscheid, J.; et al. HTLV-I and Strongyloides in Australia: The worm lurking beneath. Adv. Parasitol. 2021, 111, 119-201. [CrossRef]

3. Bisoffi, Z.; Buonfrate, D.; Montresor, A.; Requena-Mendez, A.; Munoz, J.; Krolewiecki, A.J.; Gotuzzo, E.; Mena, M.A.; Chiodini, P.L.; Anselmi, M.; et al. Strongyloides stercoralis: A plea for action. PLoS Negl. Trop. Dis. 2013, 7, e2214. [CrossRef]

4. Page, W.; Judd, J.A.; Bradbury, R.S. The Unique Life Cycle of Strongyloides stercoralis and Implications for Public Health Action. Trop. Med. Infect. Dis. 2018, 3, 53. [CrossRef] [PubMed]

5. Einsiedel, L.; Fernandes, L. Strongyloides stercoralis: A cause of morbidity and mortality for indigenous people in Central Australia. Intern. Med. J. 2008, 38, 697-703. [CrossRef] [PubMed]

6. Miller, A.; Smith, M.L.; Judd, J.A.; Speare, R. Strongyloides stercoralis: Systematic review of barriers to controlling strongyloidiasis for Australian indigenous communities. PLoS Negl. Trop. Dis. 2014, 8, e3141. [CrossRef] [PubMed]

7. Carvalho, E.M.; Da Fonseca Porto, A. Epidemiological and clinical interaction between HTLV-1 and Strongyloides stercoralis. Parasite Immunol. 2004, 26, 487-497. [CrossRef]

8. Kassalik, M.; Monkemuller, K. Strongyloides stercoralis hyperinfection syndrome and disseminated disease. Gastroenterol. Hepatol. 2011, 7, 766-768.

9. Marcos, L.A.; Terashima, A.; Dupont, H.L.; Gotuzzo, E. Strongyloides hyperinfection syndrome: An emerging global infectious disease. Trans. R. Soc. Trop. Med. Hyg. 2008, 102, 314-318. [CrossRef]

10. Keiser, P.B.; Nutman, T.B. Strongyloides stercoralis in the Immunocompromised Population. Clin. Microbiol. Rev. 2004, 17, 208-217. [CrossRef]

11. Hirata, T.; Uchima, N.; Kishimoto, K.; Zaha, O.; Kinjo, N.; Hokama, A.; Sakugawa, H.; Kinjo, F.; Fujita, J. Impairment of host immune response against Strongyloides stercoralis by human T cell lymphotropic virus type 1 infection. Am. J. Trop. Med. Hyg. 2006, 74, 246-249. [CrossRef] 
12. Satoh, M.; Toma, H.; Sugahara, K.; Etoh, K.-I.; Shiroma, Y.; Kiyuna, S.; Takara, M.; Matsuoka, M.; Yamaguchi, K.; Nakada, K.; et al. Involvement of IL-2/IL-2R system activation by parasite antigen in polyclonal expansion of CD4 $4^{+} 25^{+}$HTLV-1-infected T-cells in human carriers of both HTLV-1 and S. stercoralis. Oncogene 2002, 21, 2466-2475. [CrossRef] [PubMed]

13. Patey, O.; Gessain, A.; Breuil, J.; Courillon-Mallet, A.; Daniel, M.T.; Miclea, J.M.; Roucayrol, A.M.; Sigaux, F.; Lafaix, C. Seven years of recurrent severe strongyloidiasis in an HTLV-I-infected man who developed adult T-cell leukaemia. AIDS 1992, 6, 575-579. [CrossRef] [PubMed]

14. Terashima, A.; Alvarez, H.; Tello, R.; Infante, R.; Freedman, D.O.; Gotuzzo, E. Treatment failure in intestinal strongyloidiasis: An indicator of HTLV-I infection. Int. J. Infect. Dis. 2002, 6, 28-30. [CrossRef]

15. Montes, M.; Sanchez, C.; Verdonck, K.; Lake, J.E.; Gonzalez, E.; Lopez, G.; Terashima, A.; Nolan, T.; Lewis, D.E.; Gotuzzo, E.; et al Regulatory T cell expansion in HTLV-1 and strongyloidiasis co-infection is associated with reduced IL-5 responses to Strongyloides stercoralis antigen. PLoS Negl. Trop. Dis. 2009, 3, e456. [CrossRef] [PubMed]

16. Einsiedel, L.; Pham, H.; Talukder, M.R.; Taylor, K.; Wilson, K.; Kaldor, J.; Gessain, A.; Woodman, R. Very high prevalence of infection with the human T cell leukaemia virus type 1c in remote Australian Aboriginal communities: Results of a large cross-sectional community survey. PLoS Negl. Trop. Dis. 2021, 15, e0009915. [CrossRef] [PubMed]

17. Kearns, T.M.; Currie, B.J.; Cheng, A.C.; McCarthy, J.; Carapetis, J.R.; Holt, D.C.; Page, W.; Shield, J.; Gundjirryirr, R.; Mulholland, E.; et al. Strongyloides seroprevalence before and after an ivermectin mass drug administration in a remote Australian Aboriginal community. PLoS Negl. Trop. Dis. 2017, 11, e0005607. [CrossRef] [PubMed]

18. Einsiedel, L.J.; Pham, H.; Woodman, R.J.; Pepperill, C.; Taylor, K.A. The prevalence and clinical associations of HTLV-1 infection in a remote Indigenous community. Med. J. Aust. 2016, 205, 305-309. [CrossRef]

19. Einsiedel, L.; Spelman, T.; Goeman, E.; Cassar, O.; Arundell, M.; Gessain, A. Clinical associations of Human T-Lymphotropic Virus type 1 infection in an indigenous Australian population. PLoS Negl. Trop. Dis. 2014, 8, e2643. [CrossRef]

20. Alemu, M.; Kinfe, B.; Tadesse, D.; Mulu, W.; Hailu, T.; Yizengaw, E. Intestinal parasitosis and anaemia among patients in a Health Center, North Ethiopia. BMC Res. Notes 2017, 10, 632. [CrossRef]

21. Hays, R.; Esterman, A.; Giacomin, P.; Loukas, A.; McDermott, R. Does Strongyloides stercoralis infection protect against type 2 diabetes in humans? Evidence from Australian Aboriginal adults. Diabetes Res. Clin. Pract. 2015, 107, 355-361. [CrossRef]

22. Australian Bureau of Statistics. Themes: QuickStats. Available online: https://www.abs.gov.au/websitedbs/censushome.nsf/ home/quickstats?opendocument\&navpos=220 (accessed on 21 March 2020).

23. Northern Territory Government. BushTel. Available online: https:/ / bushtel.nt.gov.au/search (accessed on 24 August 2020).

24. Talley, N.J.; O'Connor, S. Clinical Examination: A Systematic Guide to Physical Diagnosis; Elsevier Health Sciences: Amsterdam NSW, Australia, 2014.

25. Einsiedel, L.; Pham, H.; Talukder, M.R.R.; Liddle, J.; Taylor, K.; Wilson, K.; Jersmann, H.; Gessain, A.; Woodman, R.; Kaldor, J. Pulmonary Disease Is Associated with Human T-Cell Leukemia Virus Type 1c Infection: A Cross-sectional Survey in Remote Aboriginal Communities. Clin. Infect. Dis. 2021, 73, e1498-e1506. [CrossRef] [PubMed]

26. Australian Bureau of Statistics. Socio-Economic Advantage and Disadvantage. Available online: https://www.abs.gov.au/ ausstats/abs@.nsf/Lookup/by\%20Subject/2071.0 \{\}2016 \{\}Main\%20Features \{\}Socio-Economic\%20Advantage\%20and\%20 Disadvantage \{\} 123 (accessed on 24 August 2020).

27. Hays, R.; Esterman, A.; McDermott, R. Control of chronic Strongyloides stercoralis infection in an endemic community may be possible by pharmacological means alone: Results of a three-year cohort study. PLoS Negl. Trop. Dis. 2017, 11, e0005825 [CrossRef] [PubMed]

28. Paltridge, M.; Smith, S.; Traves, A.; McDermott, R.; Fang, X.; Blake, C.; Milligan, B.; D’Addona, A.; Hanson, J. Rapid Progress toward Elimination of Strongyloidiasis in North Queensland, Tropical Australia, 2000-2018. Am. J. Trop. Med. Hyg. 2020, 102, 339-345. [CrossRef] [PubMed]

29. Forrer, A.; Khieu, V.; Schindler, C.; Schär, F.; Marti, H.; Char, M.C.; Muth, S.; Odermatt, P. Ivermectin Treatment and Sanitation Effectively Reduce Strongyloides stercoralis Infection Risk in Rural Communities in Cambodia. PLoS Negl. Trop. Dis. 2016, 10, e0004909. [CrossRef]

30. Miller, A.; Young, E.L.; Tye, V.; Cody, R.; Muscat, M.; Saunders, V.; Smith, M.L.; Judd, J.A.; Speare, R. A Community-Directed Integrated Strongyloides Control Program in Queensland, Australia. Trop. Med. Infect. Dis. 2018, 3, 48. [CrossRef]

31. Hansen, M.; Bowden, E.; Currie, B.J.; Ward, L.; Andrews, R.M.; Dhurrkay, R.G.; Gurruwiwi, G.G.; Kearns, T. Strongyloides stercoralis seropositivity is not associated with increased symptoms in a remote Aboriginal community. Intern. Med. J. 2021, 51, 1286-1291. [CrossRef]

32. World Health Organization. Strongyloidiasis. 2020. Available online: https://www.who.int/intestinal_worms/epidemiology/ strongyloidiasis/en/ (accessed on 24 August 2020).

33. Tracey, E.F.; McDermott, R.A.; McDonald, M.I. Do worms protect against the metabolic syndrome? A systematic review and meta-analysis. Diabetes Res. Clin. Pract. 2016, 120, 209-220. [CrossRef]

34. Chordia, P.; Christopher, S.; Abraham, O.; Muliyil, J.; Kang, G.; Ajjampur, S. Risk factors for acquiring Strongyloides stercoralis infection among patients attending a tertiary hospital in south India. Indian J. Med. Microbiol. 2011, 29, 147-151. [CrossRef]

35. McGuire, E.; Welch, C.; Melzer, M. Is Strongyloides seropositivity associated with diabetes mellitus? A retrospective case-control study in an East London NHS Trust. Trans. R. Soc. Trop. Med. Hyg. 2018, 113, 189-194. [CrossRef] 
36. Rajamanickam, A.; Munisankar, S.; Bhootra, Y.; Dolla, C.; Thiruvengadam, K.; Nutman, T.B.; Babu, S. Metabolic Consequences of Concomitant Strongyloides stercoralis Infection in Patients with Type 2 Diabetes Mellitus. Clin. Infect. Dis. 2019, 69, 697-704. [CrossRef] [PubMed]

37. Rajamanickam, A.; Munisankar, S.; Thiruvengadam, K.; Menon, P.A.; Dolla, C.; Nutman, T.B.; Babu, S. Impact of Helminth Infection on Metabolic and Immune Homeostasis in Non-diabetic Obesity. Front. Immunol. 2020, 11, 2195. [CrossRef] [PubMed]

38. Silas, E.; Ndlovu, S.; Tshilwane, S.I.; Mukaratirwa, S. Immunological and Pathophysiological Outcomes of Helminth Infections and Type 2 Diabetes Comorbidity Studies in Humans and Experimental Animals-A Scoping Review. Appl. Sci. 2021, 11, 8079. [CrossRef]

39. Lontchi-Yimagou, E.; Sobngwi, E.; Matsha, T.E.; Kengne, A.P. Diabetes Mellitus and Inflammation. Curr. Diabetes Rep. 2013, 13, 435-444. [CrossRef]

40. Mendonça, S.C.; Gonçalves-Pires, M.d.R.F.; Rodrigues, R.M.; Ferreira, A., Jr.; Costa-Cruz, J.M. Is there an association between positive Strongyloides stercoralis serology and diabetes mellitus? Acta Trop. 2006, 99, 102-105. [CrossRef]

41. Arifin, N.; Hanafiah, K.M.; Ahmad, H.; Noordin, R. Serodiagnosis and early detection of Strongyloides stercoralis infection. J. Microbiol. Immunol. Infect. 2019, 52, 371-378. [CrossRef]

42. Bisoffi, Z.; Buonfrate, D.; Sequi, M.; Mejia, R.; Cimino, R.O.; Krolewiecki, A.J.; Albonico, M.; Gobbo, M.; Bonafini, S.; Angheben, A.; et al. Diagnostic accuracy of five serologic tests for Strongyloides stercoralis infection. PLoS Negl. Trop. Dis. 2014, 8, e2640. [CrossRef]

43. Requena-Méndez, A.; Buonfrate, D.; Gomez-Junyent, J.; Zammarchi, L.; Bisoffi, Z.; Muñoz, J. Evidence-Based Guidelines for Screening and Management of Strongyloidiasis in Non-Endemic Countries. Am. J. Trop. Med. Hyg. 2017, 97, 645-652. [CrossRef]

44. Kalantari, N.; Chehrazi, M.; Ghaffari, S.; Gorgani-Firouzjaee, T. Serological assays for the diagnosis of Strongyloides stercoralis infection: A systematic review and meta-analysis of diagnostic test accuracy. Trans. R. Soc. Trop. Med. Hyg. 2020, 114, 459-469. [CrossRef]

45. Buonfrate, D.; Sequi, M.; Mejia, R.; Cimino, R.O.; Krolewiecki, A.J.; Albonico, M.; Degani, M.; Tais, S.; Angheben, A.; RequenaMendez, A.; et al. Accuracy of five serologic tests for the follow up of Strongyloides stercoralis infection. PLoS Negl. Trop. Dis. 2015, 9, e0003491. [CrossRef]

46. Carrilho, G.F.; DaCosta, G.M.; Olivi, M.J.; Vicentini, V.; Anibal, F.d.F. Anemia in patients with intestinal parasitic infections. Rev. Ibero-Latinoam. Parasitol. 2011, 70, 206-211.

47. Paltridge, M.; Traves, A. The health effects of strongyloidiasis on pregnant women and children: A systematic literature review. Trop. Med. Infect. Dis. 2018, 3, 50. [CrossRef] [PubMed]

48. Wilson, A.; Fearon, D. Paediatric strongyloidiasis in central Australia. Trop. Med. Infect. Dis. 2018, 3, 64. [CrossRef] [PubMed]

49. Waller, K.C.F.; Chew, R.; Tsai, D.; Einsiedel, L. Pulmonary strongyloidiasis presenting as infective exacerbation of bronchiectasis: A case report from central Australia. In Proceedings of the Update from the HOT NORTH. ASID Annual Scientific Meeting, Darwin, Australia, 16-18 May 2019 